\title{
Performance analysis of wireless-powered cognitive radio networks with ambient backscatter
}

\author{
Daniyal Munir ${ }^{1}$, Syed Tariq Shah ${ }^{1,2}$, Kae Won Choi $^{1}$, Tae-Jin Lee ${ }^{1}$ and Min Young Chung ${ }^{1 *}$ (D)
}

\begin{abstract}
Ambient backscatter is a promising wireless communication technique where low-power users communicate with each other without any dedicated power source. These communicating users transmit their information by reflecting ambient radio-frequency (RF) signals. In this paper, we propose an ambient backscatter communications-assisted wireless-powered underlay cognitive radio network (CRN). The proposed CRN consists of a single primary transmitter (PT) and multiple primary receivers (PRs), secondary transmitters (STs), and secondary receivers (SRs). For efficient utilization of radio resources, the STs in the proposed scheme dynamically adopt either harvest-then-transmit mode or backscatter mode. Furthermore, PRs cooperate with STs to select an appropriate mode for their communication with SRs. To evaluate the performance of our proposed scheme, we conduct system-level simulations. Numerical results show that the performance of the secondary system can be improved in terms of throughput with minimum effect on the communication of primary users.
\end{abstract}

Keywords: Ambient backscatter, Underlay cognitive radio, Harvest-then-transmit

\section{Introduction}

For the realization of Internet of things (IoT), low-power wireless sensor networks (WSNs) have been adopted in numerous applications, such as health care, traffic control, surveillance, and so on. In general, WSNs operate in the unlicensed industrial, scientific, and medical (ISM) spectrum [1]. However, with the extensive use of Wi$\mathrm{Fi}$, ZigBee, and Bluetooth in modern day applications, the mutual interference problem has become more challenging [2]. To alleviate this problem for a large number of sensors, cognitive radio technology was incorporated in WSNs, which yields cognitive radio sensor networks (CRSNs) [3]. Sensors, in a CRSN, can exploit more channel access opportunities in an underutilized licensed spectrum.

In CRSNs, secondary users can share the spectrum with primary users by adopting one of the two known modes: overlay and underlay modes. In overlay mode, secondary

\footnotetext{
*Correspondence: mychung@skku.edu

'Department of Electrical and Computer Engineering, Sungkyunkwan University, 2066 Seobu-Ro, Jangan-Gu, 16419 Suwon, Gyeonggi-Do, South Korea

Full list of author information is available at the end of the article
}

users can access radio resources (i.e., channels) owned by the primary network only if the primary channels are idle. However, for some wireless broadcast services such as TV and FM radio, the primary channels may be busy most of the time and secondary users may be unable to access these channels opportunistically. On the other hand, in underlay mode, secondary users can concurrently access the channels owned by the primary network, provided that the resulted interference at primary users remains below a predefined threshold value [4]. Operating on this mode may become challenging for secondary users in densely deployed area since these users will cause more interference to closely located primary users. Therefore, we need to find substitute techniques to solve this problem and enhance the performance of the secondary system.

In order to enable opportunistic communication between secondary transmitters (STs) and secondary receivers (SRs), ambient backscatter communication was proposed recently [5]. Ambient backscatter users operate on the principle of reflecting received radio frequency (RF) signals from an ambient source such as TV tower, WiFi access point (AP), and cellular base station [6].
Springer Open

(c) The Author(s). 2019 Open Access This article is distributed under the terms of the Creative Commons Attribution 4.0 International License (http://creativecommons.org/licenses/by/4.0/), which permits unrestricted use, distribution, and reproduction in any medium, provided you give appropriate credit to the original author(s) and the source, provide a link to the Creative Commons license, and indicate if changes were made. 
Note that instantaneous excitation signals provide enough power to a backscatter user to carry out its operations [7]. Hence, a dedicated time for energy harvesting is not required.

From the perspective of primary network, the backscattered (reflected) signals received at the primary receiver (PR) are treated as multipath signals from the primary transmitter (PT). This is because of the fact that the modulation rate of backscatter is very low as compared to the modulation rate of the PT's transmission. Hence, the backscattered signals are treated as constant signals from the viewpoint of a PR. Moreover, PR can prevent the effect of multipath distortion from backscattering users by implementing existing techniques used in modern wireless networks, such as cyclic prefix in OFDMA networks [5]. Therefore, ambient backscatter communications with its ultra low-power and low-complexity characteristics can be conveniently employed in a CRSN.

Recently, RF energy harvesting has been introduced and implemented in CRSNs [8]. This leads to a new type of networks, called wireless-powered CRSNs. In this network, the STs are able to harvest energy from primary signals, and use the harvested energy to transfer data to their SRs through a primary channel. Therefore, the transmission used in wireless-powered CRSN is known as harvestthen-transmit mode [9]. A wireless-powered CRSN can be integrated with ambient backscatter communication to improve the performance of the secondary system. Two possible communication modes can be adopted in such networks: (i) harvest-then-transmit mode and (ii) ambient backscatter mode [10]. However, integrating ambient backscatter communications with underlay CRSN may raise different issues as compared to overlay CRSN model considered in [10]. Some of the major challenges faced in underlay CRSN are as follows: (a) how can a secondary user choose between backscatter mode or harvest-thentransmit mode; (b) while using harvest-then-transmit mode, how much time is used for harvesting energy and information transmission; and (c) how to estimate the interference at the PRs.

In order to address the above mentioned issues, we use ambient backscatter-assisted wireless-powered network proposed by [10] in an underlay environment. The proposed CRN consists of a PT and multiple PRs, STs, and SRs. All PRs, STs, and SRs have the capability of performing RF communication and/or ambient backscatter communication. STs considered in our proposed network are energy-constraint devices and harvest energy from ambient RF signals. Thus, STs transmit their information using either harvest-then-transmit mode or backscatter mode. STs select one of the two modes based on the estimated interference they may cause to nearby PRs. STs estimate this interference with the help of channel state information (CSI) between PT and PRs, which is reported by PRs using backscatter communication. The main contributions of this paper are as follows:

- We propose a transmission mode selection mechanism for secondary users, where STs can select either harvest-then-transmit mode or ambient backscatter mode based on the estimated interference at PRs.

- In order to estimate the interference at the PR, we propose an ambient backscattering-based CSI reporting mechanism. PRs report their CSI to nearby STs using backscatter communication.

- To evaluate the performance of the proposed scheme, we conduct system-level simulations. The numerical analysis shows that the proposed scheme improves the performance of the secondary system in terms of average data rates with a minimal effect on the primary communications average data rates.

A preliminary version of this work has been presented at a conference, which briefly describes the integration of ambient backscatter with underlay CRSN [11].

The rest of this paper is organized as follows. In the next section, we present related works. System model and problem formulation are introduced in Section 3. Section 4 explains the proposed scheme in details. Performance of the proposed solution is evaluated in Section 5 and finally, we conclude this paper in the last section.

\section{Related work}

In CRSN, the battery life of a sensor user is very important, because it highly affects the network topology once the battery runs out. To improve the network lifetime and energy efficiency, a lot of effort has been put together by the research society [12-16]. Nonetheless, battery replacement is still required to keep the CRSN active. However, changing the batteries usually costs a lot and can even be dangerous where the sensors are used for monitoring radioactive and toxic materials. Instead of replacing the batteries, wireless power transfer with simultaneous information transfer (SWIPT) was first proposed by Varshney [17]. RF energy transfer was further studied for WSNs in [18-20].

In CRSN, despite reliable channel state between PT and secondary users, there would be no gain if the secondary users experience lack of energy. This motivated [21] to introduce the RF energy harvesting to the cooperative relaying CRSN. The users can cooperate at both information and energy levels, through which the energy-limited secondary users can operate continuously. In a similar environment, the authors in [22] proposed an optimal resource allocation scheme for secondary users to maximize the sum throughput. However, for wireless-powered CRSNs, when the secondary users have less opportunity 
to access the primary channels, backscatter communications can be used to improve the low overall transmission rate for secondary networks [10].

Recently, backscatter communications powered by RF energy harvesting has received a lot of attention. Parks et al. in [23] proposed a coding mechanism for backscatter users that enables long range communication between them as well as improves the transmission rate as high as $1 \mathrm{Mbps}$. To provide Internet connectivity for these low power devices, authors in [24] reuse existing Wi-Fi infrastructure to demonstrate its feasibility. Authors in [6] have proposed a network architecture where two users can communicate with each other by integrating energy harvesting and backscatter communications. They consider a scenario where power beacons are deployed for wirelessly powering these users. The users transmit data to their potential receiver by modulating and reflecting a portion of received signals from the power beacons. However, deploying power beacons to recharge an enormous amount of low power users may prove to be costly.

Backscatter communications was also addressed in some recent works $[7,25,26]$. To enhance the throughput of backscatter communications, authors in [25] proposed a reader design with multi-antenna wireless energy beamforming for multiple backscatter users. Lyu et al. [7] have proposed resource allocation policies for multiuser backscatter communication systems. They have considered a dedicated reader antenna, which excites the backscatter user for their concurrent communication with the reader. Authors in [26] have proposed a wireless powered communications network assisted by backscatter communications. Their model consists of a power station, an information receiver and multiple users that can work in either backscatter mode or harvest-thentransmit mode. These works provide useful insight into the backscatter communications and, however, require special infrastructure to generate the excitation RF signals that backscatter users can reflect. Therefore, ambient backscatter communications should be given more attention to exploring cost-effective possibilities for low-power devices.

Recent research works such as [10] have reduced the need for specialized infrastructure. In [10], an ambient backscatter-assisted CRSN was proposed, where two types of users are present in the network, which can operate in backscatter and harvest-then-transmit modes. For flexible network deployment, the authors assumed that a user could operate in one of the two modes. A singleuser CRSN was considered, and a tradeoff between the backscatter and harvest-then-transmit modes was analyzed. Based on [10], the authors in [27] extended the work for a multi-user case and studied the optimal time allocation policy. In [10] and [27], authors have studied an overlay CRSN to maximize the secondary system throughput. However, in practical systems, the possibility of finding idle channels is very low for overlay-based CRSN, which motivates us to study this scenario in an underlay CRSN.

\section{System model}

As shown in Fig. 1, we consider an underlay CRSN scenario, where energy-constrained secondary users simultaneously access the licensed spectrum to transmit their own data. The network consists of primary users, primary

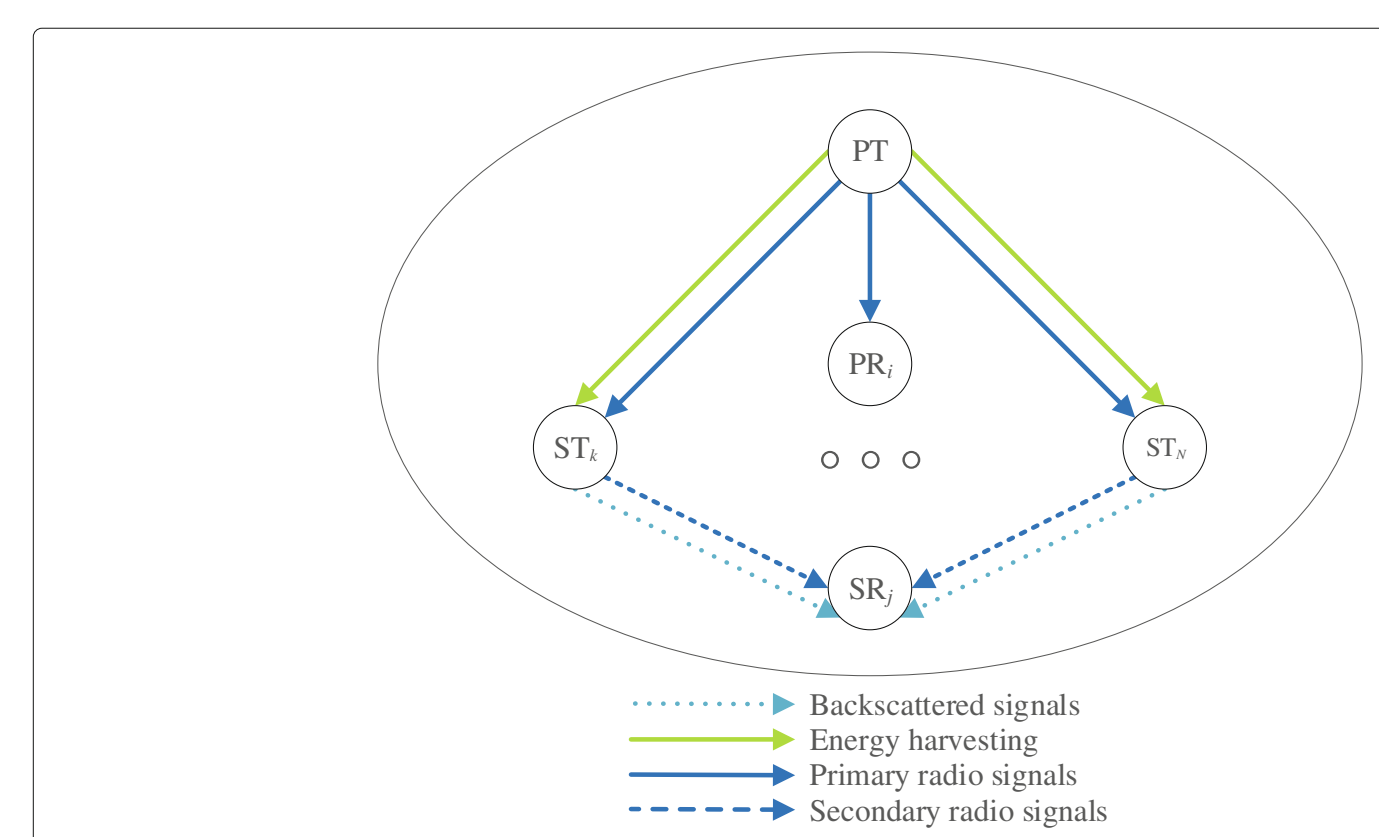

Fig. 1 System model for the proposed scheme 
transmitter (PT) and primary receiver (PR), and secondary users, secondary transmitter (ST) and secondary receiver (SR). We denote set of PRs, set of SRs, and set of STs as $\mathcal{I}, \mathcal{J}$ and $\mathcal{K}$, respectively, where $\mathcal{I}=\{1,2,3, \cdots, L\}$, $\mathcal{J}=\{1,2,3, \cdots, M\}$ and $\mathcal{K}=\{1,2,3, \cdots, N\}$. The PRs and SRs are randomly deployed within the disc radius of PT using Poisson point process (PPP) with densities $\lambda_{p r}$ and $\lambda_{s r}$, respectively. Then, STs are deployed randomly using PPP within the radius of each SR with density $\lambda_{s t}$.

The PT continuously broadcasts radio signals within its coverage area to transfer information to PRs. PRs receive these broadcast signals using their RF communication interface. Furthermore, PRs concurrently report CSI between PT and PR to STs located nearby, using their backscatter interface. Those STs that will receive the CSI from a PR are considered as its nearby STs. Based on the information received from the PR, nearby STs estimate the interference that may be induced to the PR. This estimated interference is then used by the STs to select either harvest-then-transmit mode or backscatter mode. It should be noted that STs cannot operate on harvest-then-transmit mode and backscatter mode simultaneously [5]. Therefore, STs switch between one of the two modes to transfer their information to SRs. To receive useful information from STs, SRs may tune to either RF communication or backscatter communication, depending upon the communication mode used by STs.

For the harvest-then-transmit mode, STs rely only on harvested energy from RF signals transmitted by PT and store the harvested energy in their energy storage units for further data transmission. On the other hand, when the backscatter mode is activated, the STs can backscatter the modulated PT signals to the SRs instantaneously. It is assumed that SRs have the perfect knowledge of each ST's operating mode and are able to select the corresponding demodulators for effective communication [10]. Moreover, the channel gains between the users are modeled by the quasi-static block-fading process. In other words, the channel remains constant over the block time $T$, but may vary independently from one block to another following an identical distribution. In addition, the small-scale fading experienced by these channels is represented by Rayleigh fading model.

The transmission block structure of the proposed scheme is shown in Fig. 2. A time block in which PT transmits signals to PRs can be utilized by STs in two main phases: CSI reporting phase and communication phase. For $\beta T$ duration, i.e., CSI reporting phase, PRs report their CSI to nearby STs by using backscatter communication. Based on the received CSI, each ST estimates the interference it may cause to its nearby PRs. Depending on the estimated interference in the first phase, ST decides to select one of the two modes for $(1-\beta) T$ duration in communication phase.

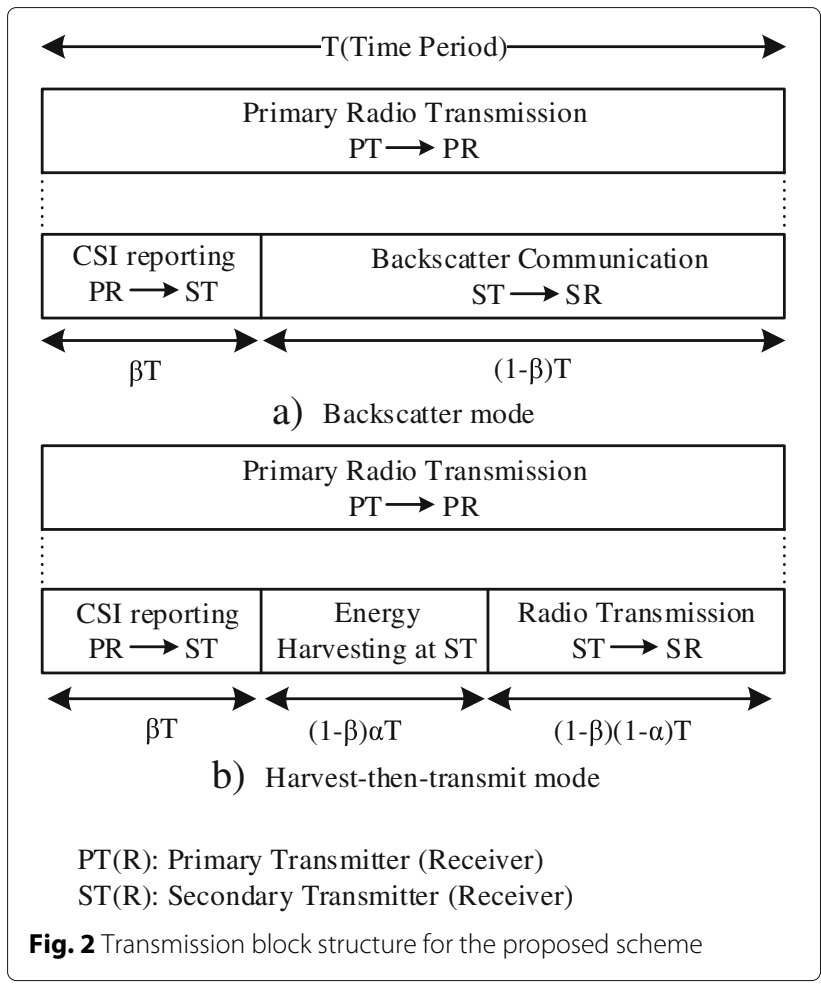

In backscatter mode, since STs do not use conventional radio components such as oscillator, mixer, and power amplifiers, they activate their backscatter module from received RF signal power. In addition, STs use the same signals to modulate their data and reflect these signals to communicate with SRs. The SRs may use either passive or active components to demodulate the backscattered signals received from STs. Figure 2a illustrates the operation for backscatter mode. STs communicate with SRs by using backscatter communications for the whole $(1-\beta) T$ duration.

In order to harvest energy and decode information simultaneously, we adopt time switching-based receiver architecture at STs [28]. In other words, STs use a portion of time for energy harvesting and use that harvested energy for radio transmission for the rest of the time. We assume that STs consume all the harvested energy to transmit the signals to SR. Figure $2 \mathrm{~b}$ shows a time switching scheme for the harvest-then-transmit mode of the proposed scheme. During this mode, the communication phase $(1-\beta) T$ is further divided into $\alpha$ and $(1-\alpha)$, denoting the time ratio between energy harvesting and radio transmission, respectively. ST harvests energy from the received signals of PT for $\alpha(1-\beta) T$ time and uses this harvested energy to transfer its information to SR through radio transmission for $(1-\alpha)(1-\beta) T$ time. The details of the proposed procedure are described in the following sections. 


\section{Proposed scheme}

In this section, we explain the proposed scheme for underlay CRSN assisted by backscatter communications. Figure 3 presents a graphical overview of the proposed scheme. In step 1, PT broadcasts signals to all the users located within its coverage area. PRs backscatter the received signals to report their CSI to nearby STs in step 2 . Based on the received CSI, an ST estimates interference it may cause to the nearby PRs in step 3. ST takes a decision to choose one of the two communication modes based on the estimated interference. If the interference is less than a certain threshold, in step 4a, ST chooses to harvest energy from PT signal power and transmits its information to SR using the harvested energy. On the other hand, if the estimated interference is greater than a threshold, ST chooses to communicate with the SR using backscatter communications in step $4 \mathrm{~b}$. The detailed procedure is explained in the following subsections.

\subsection{CSI reporting phase}

In conventional underlay CRSNs, PRs do not assist STs to estimate the channel conditions. This problem is more intractable for broadcast networks, where receivers do not send any acknowledgment of the reception of their data. However, authors in [29] have considered that primary users cooperate with secondary users to enhance mutual performance. In order to maintain high-performance gains for STs and minimize interference at PRs, STs should continuously monitor channel gains between PT and PRs [30]. If an ST can determine the information about the received signal strength at a $P R$, it will be able to estimate the interference it may cause to that PR. These channel gains can be estimated by using pilot-aided approaches or by employing sensors near all the PRs [30]. Therefore, for reliable estimation of interference at PRs, we assume that each PR is equipped with a backscatter interface, instead of a separate sensor, which is only used for reporting the channel conditions between PR and PT to its nearby STs. This channel condition information can then be used by the nearby STs to estimate interference which may be induced to the PR. Due to low power consumption and simple communications mechanism of backscatter communications, it is rational to assume that PRs use backscatter communications for reporting channel condition to nearby STs.

In broadcast networks such as TV transmissions, the transmissions periodically encode specific symbols for synchronization [31]. These special symbols are used by the receivers to synchronize the timing and compute different channel characteristics such as multipath. Once a PR is synchronized, it reports CSI between PR and PT to its nearby STs using its backscatter interface for $\beta T$ time. In case more than one PRs are located near a ST, there will be collision if both the PRs report CSI simultaneous. To avoid this collision, $\beta T$ time slot is further divided into $X$ sub-slots. In each slot, independent of others, a

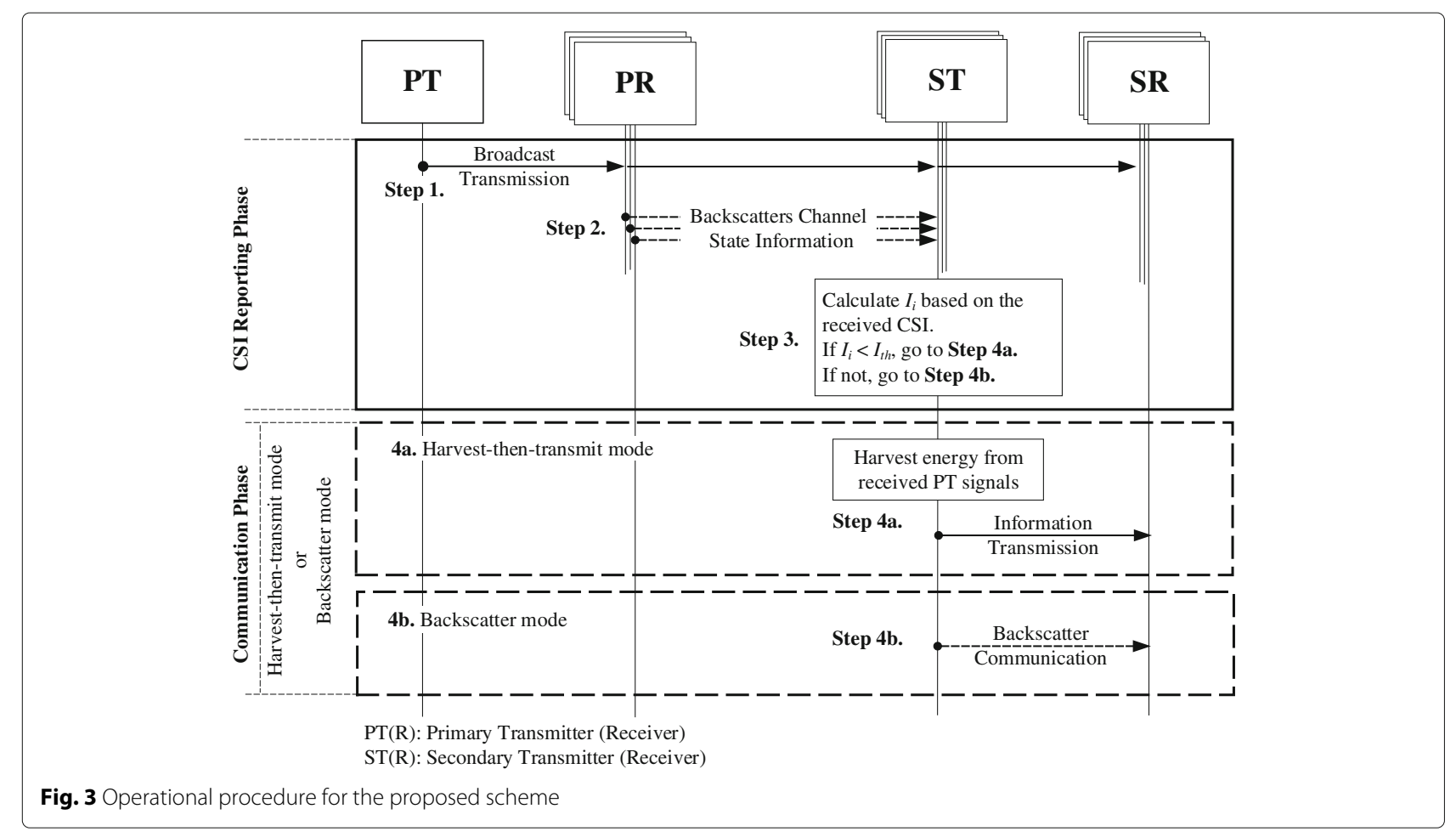


backscattering PR randomly selects a single sub-slot to transmit its signal. This divides each slot into a backscatter phase and a waiting phase of durations $1 / X$ and $(1-1 / X)$, respectively. If two or more PRs select the same sub-slot, then there will be collision, which will be reported back by the receiving ST. Those users will then back-off for a random time and then try to send their information again by randomly selecting another sub-slot in the next time frame. We adopt this simple anti-collision method for our system, however, one can further reduce the number of collisions by adopting more complex anticollision mechanisms reported in [32].

\subsection{Communication phase}

Based on the interference estimated after the CSI phase, an ST will be allowed to operate on either harvest-thentransmit mode or backscatter mode to communicate with the associated SR. Since there are $N$ number of STs present in the network, some may cause more interference to the nearby PRs and select backscatter mode. On the other hand, some STs may select harvest-then-transmit mode if the interference caused by this mode is less then a specific threshold. The average capacity of SR is computed by averaging the individual capacity of each ST. The main objective of the proposed scheme is to improve the overall data rates of the secondary system in the communication phase, which can be mathematically represented as:

$$
C_{s r}= \begin{cases}C_{b} & , \text { Backscatter mode } \\ C_{r t} & , \text { Harvest-then-transmit mode }\end{cases}
$$

where $C_{b}$ and $C_{r t}$ are the data rates of backscattering and harvest-then-transmit, respectively. The procedure for calculating the data rates of ST using two different modes is explained in the following.

\subsubsection{Backscatter mode}

The transmission rate of backscatter communication depends on the RC circuit of the backscatter module [5]. The rate may vary according to the different settings of the circuit elements (such as resistance and capacitance of the modulator). In addition, reported data rates of ambient backscatter can range from $1 \mathrm{Kbps}$ [5] to $1 \mathrm{Mbps}$ [24]. Therefore, data rates of the backscatter communications with a fixed transmission rate can be calculated as:

$$
C_{b}=(1-\beta) T B_{b}
$$

where $B_{b}$ is the transmission rate of the backscatter mode considered for a unit time block. Note that instantaneous excitation signals received from $\mathrm{PT}$ provide enough power to the ST for carrying out backscatter operations [10]. Therefore, in Eq. (2), there is no need to consider the circuit energy consumption for the backscatter mode.

\subsubsection{Harvest-then-transmit mode}

The signals transmitted by PT with transmission power $P_{p}$ are received at $\mathrm{PR} i$ and can be given by:

$$
y_{i, p}=\frac{1}{\sqrt{d_{p, i}^{m}}} \sqrt{P_{p}} h_{p, i} x_{p}+n_{i},
$$

where $h_{p, i}$ is the channel coefficient between PT and PR, $d_{p, i}$ is the distance between the two users, and $n_{i}$ is the additive white Gaussian noise (AWGN) at PR. Similarly, the signals received from $\mathrm{PT}$ at $\mathrm{ST} k$ can be written as:

$$
y_{k, p}=\frac{1}{\sqrt{d_{p, k}^{m}}} \sqrt{P_{p}} h_{p, k} x_{p}+n_{k},
$$

where $h_{p, k}$ and $d_{p, k}$ are the channel coefficient and distance between PT and ST, respectively, and $n_{k}$ is the AWGN at ST. For energy harvesting duration $(1-\beta) \alpha T$, the ST $k$ harvests energy from the received signal power $\left(P_{0}\right)$, which can be given as:

$$
E_{H, k}=(1-\beta) \alpha T P_{0}=(1-\beta) \alpha T \eta \frac{P_{p}\left|h_{p, k}\right|^{2}}{d_{p, k}^{m}},
$$

where $\eta$ is the energy harvesting efficiency.

For radio transmission, ST $k$ utilizes harvested energy $E_{H, k}$ as a source of transmission power. Thus, for a successful transmission, $E_{H, k}$ should be greater than circuit energy consumption $E_{c}$, i.e., $E_{H, k}>E_{c}$. Substituting the value of $E_{H, k}$ from (5), the value of energy harvesting time $\alpha$ can be obtained as:

$$
\alpha>\frac{E_{c} d_{p, k}^{m}}{\eta(1-\beta) T P_{p}\left|h_{p, k}\right|^{2}} .
$$

The minimum energy harvesting time $\left(\alpha^{\prime}=\frac{E_{c} d_{p, k}^{m}}{\eta(1-\beta) T P_{p}\left|h_{p, k}\right|^{2}}\right)$ is required such that sufficient energy is acquired to activate the circuit of the ST for radio transmission. After accumulating enough energy for radio transmission, the ST transmits the signal $x_{s}$ for $(1-\beta)(1-\alpha) T$ duration and the transmitted power $P_{k}$ can be given as:

$$
P_{k}=\frac{E_{H, k}}{(1-\alpha)(1-\beta) T}=\frac{\eta \alpha P_{p}\left|h_{p, k}\right|^{2}}{(1-\alpha) d_{p, k}^{m}},
$$

where $P_{k}$ must be non-negative if $\alpha \geq \alpha^{\prime}$.

On the other hand, the PR $i$ reports its CSI to nearby STs by reflecting the received signals from PT by using its backscatter interface. All the STs that will receive this CSI are considered as nearby STs. When CSI is received at STs, they will then estimate the interference that may be caused to the nearby PRs. If nearby STs choose to transmit their information through radio transmission, signal-to-noiseplus-interference-ratio (SINR) at $i$ th PR can be estimated from (3) as: 


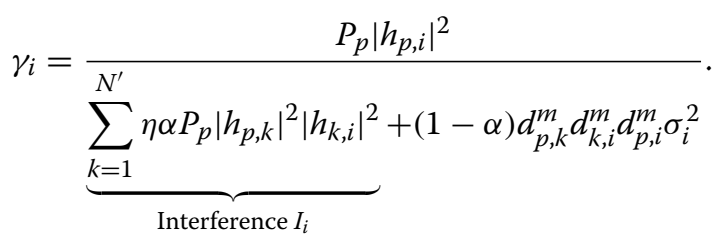

Nearby ST $k$, where $k \in\left\{1,2, \cdots, N^{\prime}\right\} \subseteq \mathcal{K}$, induce interference to PR $i$, as indicated in (8). ST $k$ should limit this interference to a certain level such that the $\operatorname{SINR}\left(\gamma_{i}\right)$ at PR $i$ does not drop below a certain threshold $\left(\gamma_{t h}\right)$. If $\gamma_{i}$ at PR $i$ is greater than $\gamma_{t h}$ (i.e. $\gamma_{i}>\gamma_{t h}$ ) after experiencing interference from ST $k$, ST $k$ will use harvest-then-transmit mode. Otherwise, if $\gamma_{i}<\gamma_{t h}$, then ST $k$ will use backscatter mode for its data transfer. The data rates of PR $i$ can be calculated as:

$$
\begin{aligned}
C_{p r} & =\mathbb{E}\left(\log _{2}\left(1+\gamma_{i}\right)\right), \\
& =\mathbb{E}\left(\log _{2}\left(1+\frac{P_{p}\left|h_{p, i}\right|^{2}}{\sum_{k=1}^{N^{\prime}} \eta \alpha P_{p}\left|h_{p, k}\right|^{2}\left|h_{k, i}\right|^{2}+(1-\alpha) d_{p, k}^{m} d_{k, i}^{m} d_{p, i}^{m} \sigma_{i}^{2}}\right)\right) .
\end{aligned}
$$

The main focus of the proposed scheme is to improve the performance of the secondary system without compromising a certain level of quality of service for the primary system. In the communication process of primary system, when PT transmits signals to PR, SR also receives those signals, which can be given as:

$$
y_{j, p}=\frac{1}{\sqrt{d_{p, j}^{m}}} \sqrt{P_{p}} h_{p, j} x_{p}+n_{j},
$$

where $h_{p, j}$ and $d_{p, j}$ are the channel coefficient and distance between PT and SR $j$, respectively, and $n_{j}$ is the AWGN at the $\mathrm{SR} j$. These signals serve as interference for $\mathrm{SR} j$. The signals received at $\mathrm{SR} j$ from $\mathrm{ST} k$ can be given as:

$$
y_{j, k}=\frac{1}{\sqrt{d_{k, j}^{m}}} \sqrt{P_{k}} h_{k, j} x_{s}+n_{j}
$$

where $h_{k, j}$ represents the channel coefficient between ST $k$ and SR $j, d_{k, j}$ is the distance between them, and $n_{j}$ is the AWGN at SR. Substituting the value of $P_{k}$ from (7) into (11), the received signal $y_{j, k}$ can be rewritten as:

$$
y_{j, k}=\frac{h_{k, j}}{\sqrt{d_{k, j}^{m}}} \sqrt{\frac{\eta \alpha P_{p}\left|h_{p, k}\right|^{2}}{(1-\alpha) d_{p, k}^{m}}} x_{s}+n_{j} .
$$

SR $j$ receives useful information from ST $k$ and interference from PT. The subsequent SINR at SR $j$ can be written as:

$$
\gamma_{j}=\frac{\eta \alpha P_{p}\left|h_{p, k}\right|^{2}\left|h_{k, j}\right|^{2} d_{p, j}^{m}}{(1-\alpha) P_{p}\left|h_{p, j}\right|^{2} d_{p, k}^{m} d_{k, j}^{m}+(1-\alpha) d_{p, j}^{m} d_{p, k}^{m} d_{k, j}^{m} \sigma_{j}^{2}},
$$

As mentioned before, ST $k$ can successfully use radio transmission only if the harvested energy is greater than the circuit energy consumption. Only then the ergodic capacity of the SR can be non-negative and can be given as:

$$
\begin{aligned}
C_{r t} & =\mathbb{E}\left((1-\beta)(1-\alpha) T \log _{2}\left(1+\gamma_{j}\right)\right), \\
& =\mathbb{E}\left((1-\beta)(1-\alpha) T \log _{2}(1+M \alpha)\right)
\end{aligned}
$$

where

$$
M=\frac{\eta P_{p}\left|h_{p, k}\right|^{2}\left|h_{k, j}\right|^{2} d_{p, j}^{m}}{(1-\alpha) P_{p}\left|h_{p, j}\right|^{2} d_{p, k}^{m} d_{k, j}^{m}+(1-\alpha) d_{p, j}^{m} d_{p, k}^{m} d_{k, j}^{m} \sigma_{j}^{2}} .
$$

\section{Performance evaluation}

\subsection{Simulation setup}

We consider a CRSN where PT is a TV broadcast tower operating on $539 \mathrm{MHz}$ frequency and a bandwidth of 6 $\mathrm{MHz}$ [5]. We consider that the PT is a source with unlimited power supply and a transmission power $P_{p}=10 \mathrm{~kW}$ [10]. All STs within the coverage area of PT, which is set as a disc of radius $1000 \mathrm{~m}$, harvest energy from the signal power of PT. We set energy harvesting efficiency, $\eta=0.6$ and path loss exponent $m=2.7$ (for urban wireless network environment). The system parameters are listed in Table 1 . The mean values of randomly generated channel gains $\left|h_{x, y}\right|^{2}, x \in\{p, k\}, y \in\{k, i, j\}$, are set to 1 .

For simplicity, the noise variance at PR, SR, and ST are assumed to be the same, i.e., $\sigma_{i}^{2}=\sigma_{j}^{2}=\sigma_{k}^{2}=0.01$. To ensure minimum effect on the communication of primary system, SINR threshold $(\gamma)$ at PR is set as $-5 \mathrm{~dB}$. The circuit power consumption is set to $-35 \mathrm{dBm}$ [10]. Unless otherwise stated, PRs and SRs are deployed in the network using PPP with densities $\lambda_{p r}$ and $\lambda_{s r}$ set to $0.2 / \mathrm{km}^{2}$ and $0.1 / \mathrm{km}^{2}$, respectively. STs are also distributed using PPP with a density $\lambda_{s t}=0.002 / \mathrm{m}^{2}$ within a radius of $50 \mathrm{~m}$ centered at each SR $j$. Therefore, in terms of distance, nearby PRs are those which are located within $50 \mathrm{~m}$ radius of STs. An example of our considered network deployment is shown in Fig. 4.

Each ST present in the simulation environment operates using the proposed scheme presented in Section 4. The capacity for both the modes is obtained from the

Table 1 Simulation parameters

\begin{tabular}{ll}
\hline Parameters & Studied value \\
\hline Energy harvesting ratio $(\alpha)$ & $0-1$ \\
CSI reporting ratio $(\beta)$ & 0.2 \\
Conversion efficiency $(\eta)$ & 0.6 \\
Coverage area (radius) of PT & 1000 meters \\
Max distance between ST and SR & 50 meters \\
Transmission power PT $\left(P_{p}\right)$ & $10 \mathrm{~kW}$ \\
Circuit power consumption & $-35 \mathrm{dBm}$ \\
Backscatter transmission rate $\left(B_{b}\right)$ & $33 \mathrm{Kbps}$
\end{tabular}




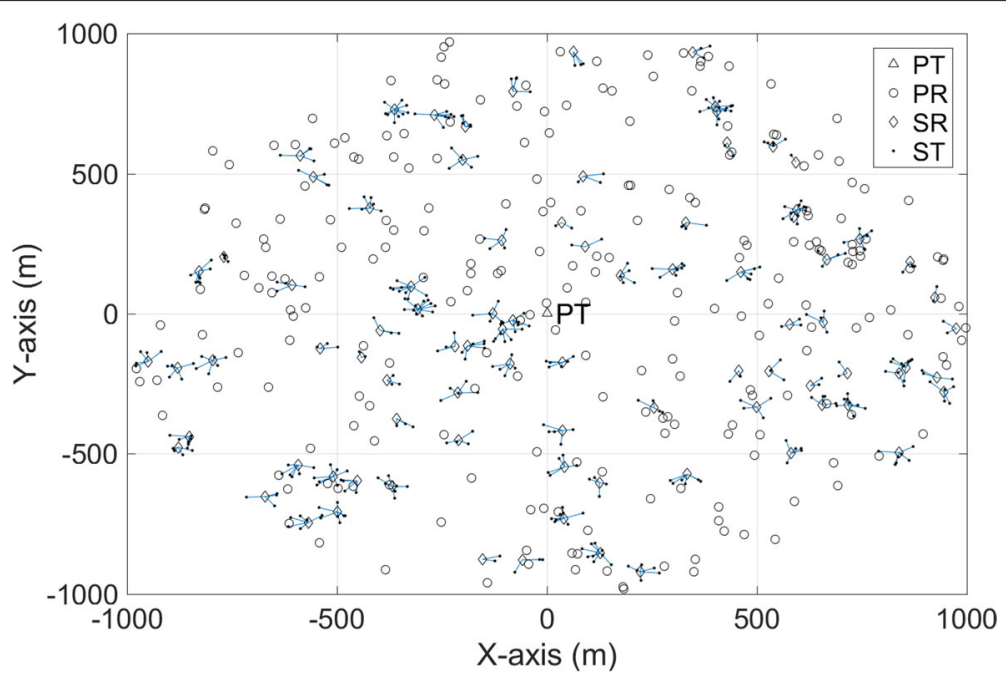

Fig. 4 The spatial distribution of an underlay CRSN modeled using PPP

equations derived in Subsection 4.2. We obtain the average capacity of the secondary system by averaging the individual capacity of each ST, operating on either backscatter mode or harvest-then-transmit mode. Other results in our simulations are also obtained from the mathematical equations presented in Section 4. All simulation results are achieved by averaging 10,000 Monte Carlo runs.

\subsection{Simulation results}

The performance of the proposed scheme is evaluated through results shown in this subsection. Different performance metrics are shown in this subsection to provide an insight to the wireless-powered underlay CRSN with backscatter communications. In the proposed scheme, when the harvest-then-transmit mode is selected, we observe that there is a tradeoff between time ratio for energy harvesting and average data rates of ST. Figure 5 shows the variation of the average data rates of ST for different values of $\alpha$. The figure plots average data rates of ST for different transmit powers of PT. As shown in the figure, the average data rate of the secondary system is low for lower values of $\alpha$ and increases as the value of $\alpha$ increases. When $\alpha>\alpha^{\prime}$, the average data rate $C_{r t}$ is concave, and it exhibits the highest value at $\alpha=0.56$. We use this value of $\alpha$ to evaluate the affect of other system

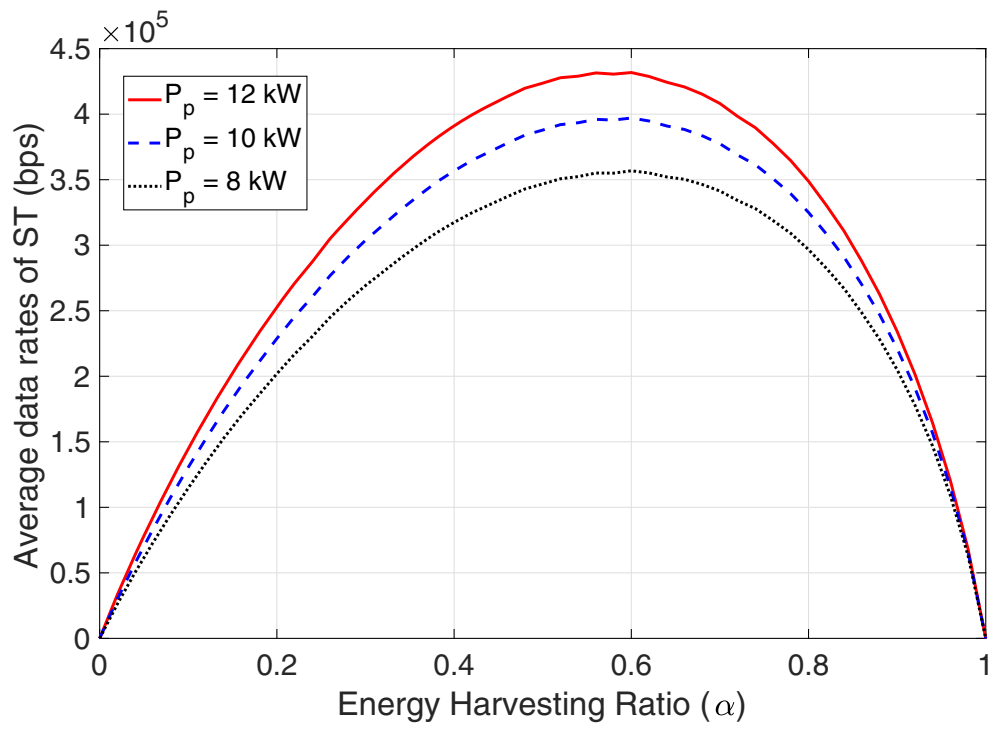

Fig. 5 Performance of the secondary system under the variation of $\alpha$, for the harvest-then-transmit mode. Other parameters: $\eta=0.6$ and $\beta=0.2$ 


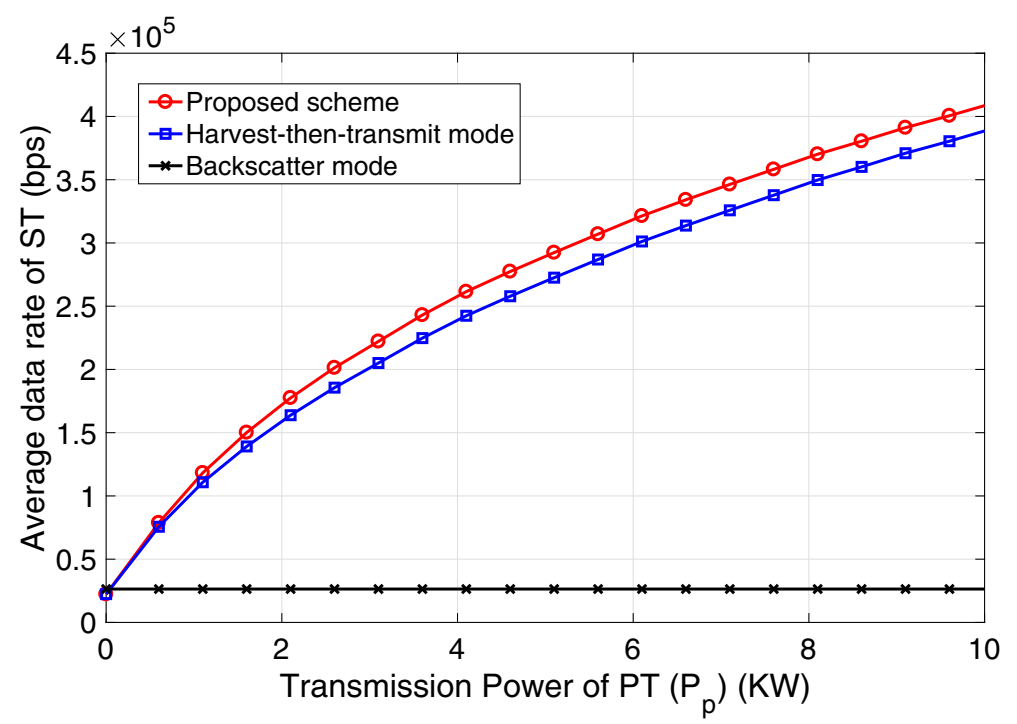

Fig. 6 Performance of the secondary system under the variation of the transmission power of PT $\left(P_{p}\right)$. Other parameters: $\alpha=0.56, \eta=0.6$ and $\beta=0.2$

parameters on the system performance. $\alpha$ represents the time spent for harvesting energy from the received signals of PT; if more energy is harvested, more power will be available for the transmission of data through radio transmission; as a result, the data rates will be improved. On the other hand, a higher value of $\alpha$ means there will be less time for radio transmission and ST will not be able to send more data which results in lower data rates.

To provide insight of the effect of the transmission power of PT $\left(P_{p}\right)$ on the proposed scheme, Fig. 6 shows the average data rate of ST for different values of $P_{p}$. For the value of $\alpha=0.56$, the figure shows that as the values of $P_{p}$ increases, the average data rate of ST increases. This is because of the fact that more energy can be harvested for higher values of $P_{p}$. The harvested energy is then used as the transmission power of ST, which results in higher data rates. It can be seen from the figure that the proposed scheme has the best performance in terms of the average data rate of ST as compared to backscatter mode and harvest-then-transmit mode.

The effect of PR density $\left(\lambda_{p r}\right)$ on the SINR of STs is shown in Fig. 7 for the proposed scheme. The figure plots the CDF of average SINR at STs for different values of $\lambda_{p r}$. Increase in the density of the PR degrades the

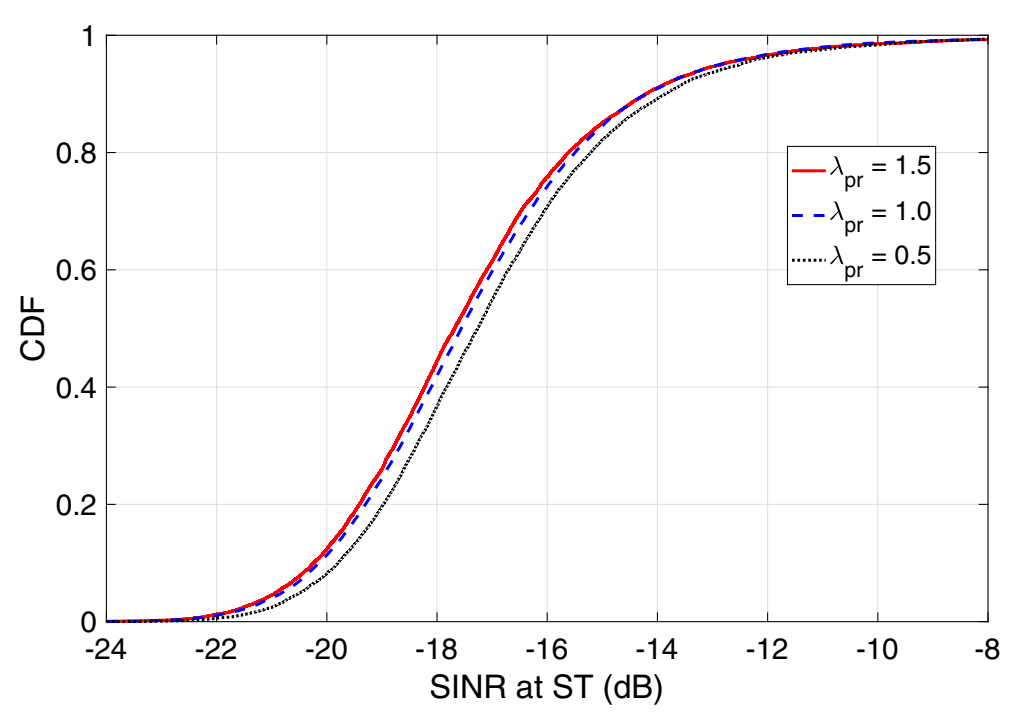

Fig. 7 Performance of the secondary system under the variation of PR density. Other parameters: $\alpha=0.56, \eta=0.6$ and $\beta=0.2$ 


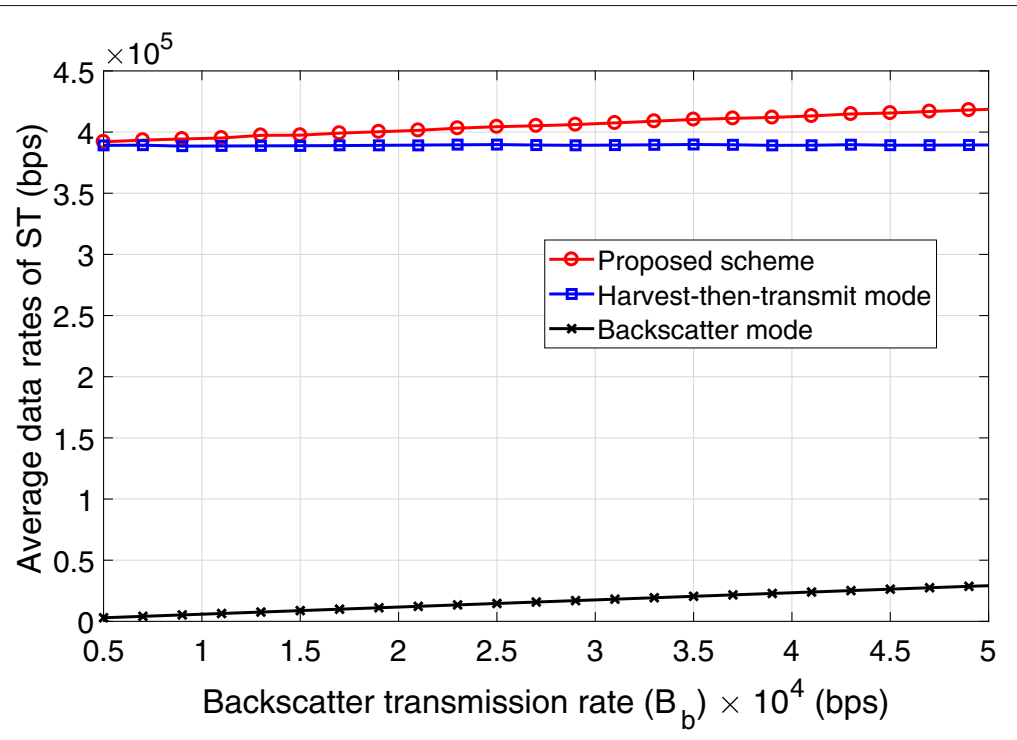

Fig. 8 Performance of the secondary system under the variation of backscatter transmission rate $\left(B_{b}\right)$. Other parameters: $\alpha=0.56, \eta=0.6$ and $\beta=0.2$

performance of STs. This is aligned with the basic concept of the underlay CRSN model because less number of STs will be able to use harvest-then-transmit mode as the density of PRs increases. Note that backscatter mode will be still used for the transmission of information from ST to SR. However, the transmission rate of backscatter mode is much less than that of the harvest-then-transmit mode, which results in the degradation of average SINR at STs.

Furthermore, we present the results to provide insight on the performance of the proposed scheme by varying different system parameters. To evaluate the performance of the secondary system, Fig. 8 shows the effect on the

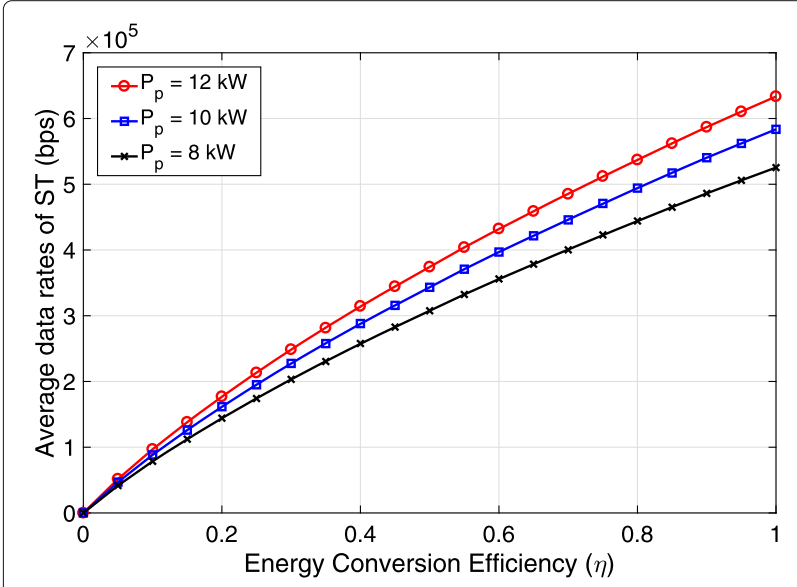

Fig. 9 Performance of the secondary system under the variation of energy harvesting efficiency $(\eta)$. Other parameters: $\alpha=0.56$, and $\beta=0.2$ average data rate of ST with the variation of backscatter transmission rate. For this performance metric, we use the value of $\alpha=0.56$ because it achieves the highest data rates for the harvest-then-transmit mode. It can be seen from the figure that the average data rate of ST achieves the best performance for the proposed scheme as compared to backscatter mode and harvest-then-transmit mode separately. This is because when the ST is unable to operate at harvest-then-transmit mode due to interference at the PR, it can use backscatter mode to transfer its information to the SR.

We then vary the energy harvesting efficiency $\eta$ shown in Fig. 9, for different values of the transmission power of PT. Average data rates of ST increases as the value of $\eta$ increases. Energy harvesting efficiency is the capability of energy harvesting circuit to convert the RF signal power into DC current. This harvested energy is then used by the ST to transmit information towards SR using active radio component. As the value of $\eta$ increases average data rate of ST also increases because ST can more efficiently harvest energy and use that energy as its transmission power. Furthermore, for higher values of $P_{p}$ the data rates increase which can be confirmed by previous figures.

For the secondary system, we finally evaluate the performance of the proposed scheme by varying the CSI reporting ratio $(\beta)$. Figure 10 shows the effect on the average data rate of $\mathrm{ST}$ with the variation of CSI reporting ratio $(\beta)$. It is intuitive that the average data rates of the secondary system will decrease with an increase in the value of $\beta$. The reason for this decrease is the direct relation of 


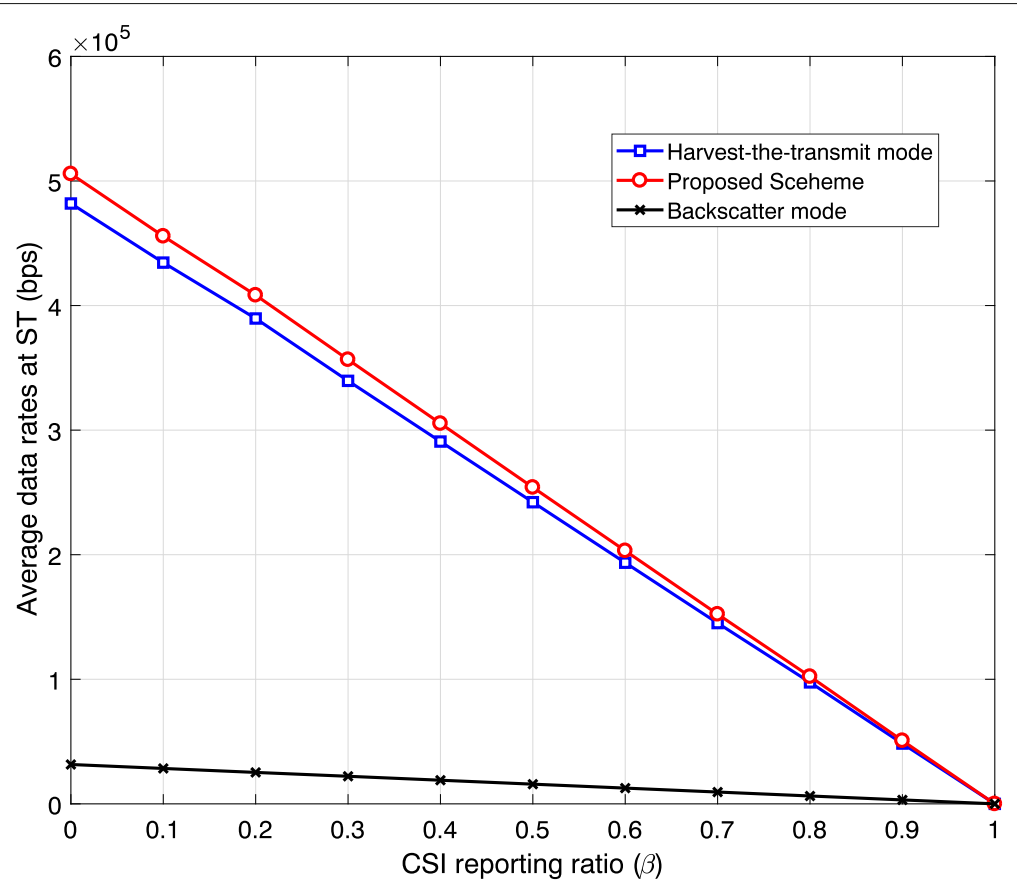

Fig. 10 Performance of the secondary system under the variation of CSI reporting ratio $(\beta)$. Other parameters: $\alpha=0.56$, and $\eta=0.6$

$\beta$ with both data rates of backscatter mode and harvestthen-transmit mode. Increasing values of $\beta$ imply that more time will be used for CSI reporting and less time will be available for communication phase.

To see the effect of energy harvesting ratio $(\alpha)$, Fig. 11 plots the average data rate of PR in the presence of STs. Since for smaller values of $\alpha$, the amount of harvested energy is less, consequently, the transmission power of
STs is also less and the interference ST may cause will be lower. On the other hand, although less time is available for transmission, STs may cause a significant interference to the nearby PRs. This is because of the fact that large number of STs are located near the PRs and the transmission power of STs is higher for higher values of $\alpha$. As a result, we choose an optimal value of $\alpha$, which maximizes average data rate of secondary

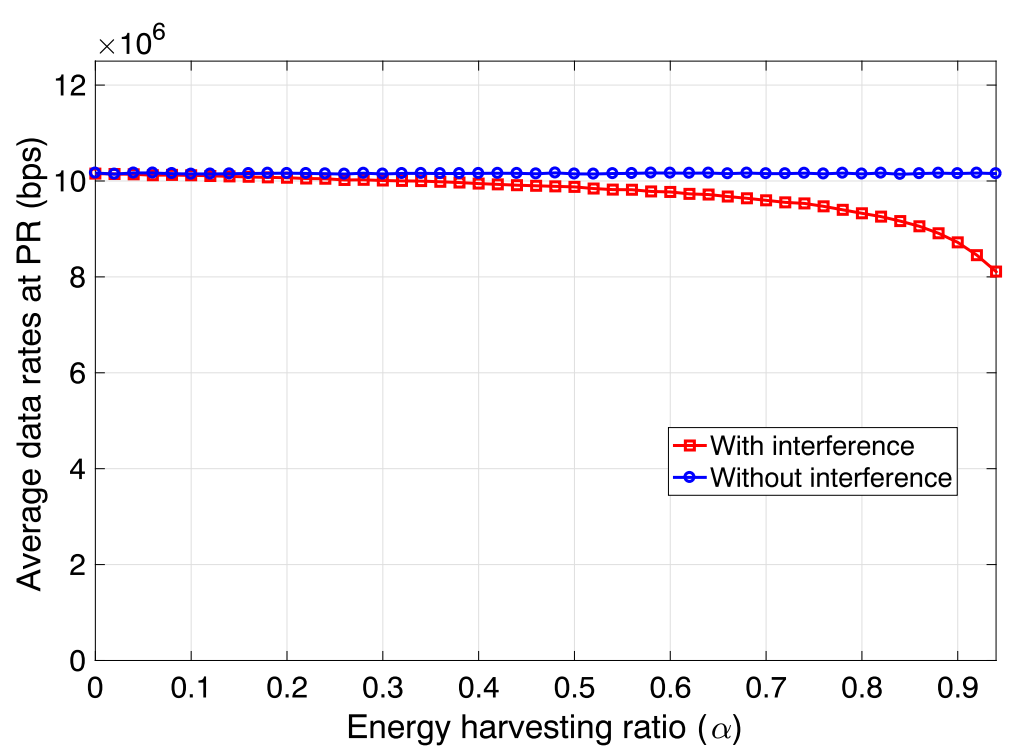

Fig. 11 Average data rate of PRs under the variation of energy harvesting ratio $(\alpha)$. Other parameters: $\gamma=-5 d B m, \eta=0.6$, and $\beta=0.2$ 


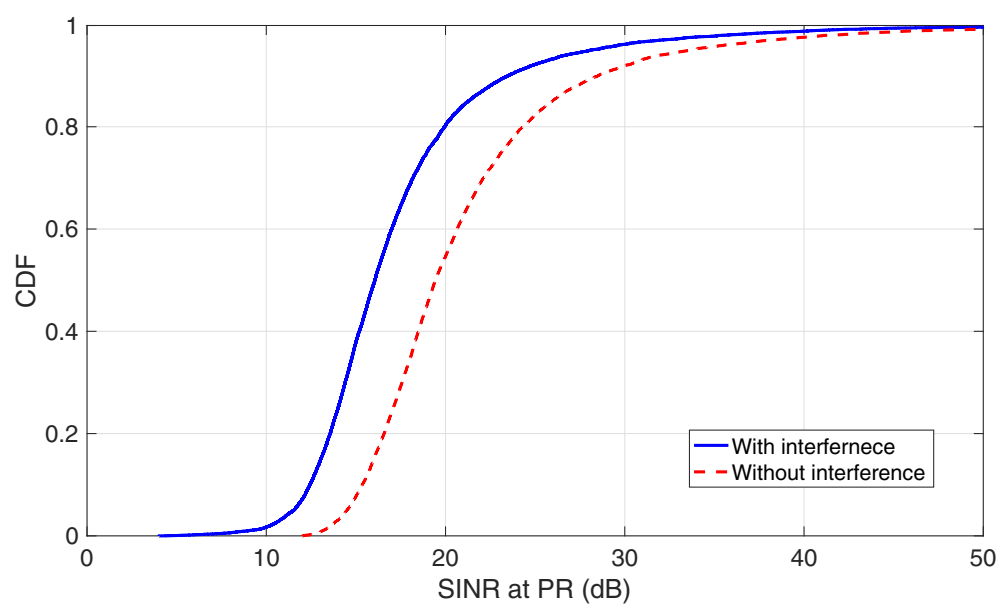

Fig. 12 Performance of primary receivers with and without secondary interference. Other parameters: $\gamma=-5 d B m, \alpha=0.56, \eta=0.6$ and $\beta=0.2$

system and minimizes the effect on the communication of primary system.

Communication of the primary system is of prime concern in CRSN and should be guaranteed a certain level of quality of service. Figure 12 plots the CDF of the average SINR at PR. The figure also shows the effect of the proposed scheme on the SINR of PR. It can be observed from the figure that the performance of $P R$ experience a slight degradation due to the presence of STs operating in underlay model. This minor degradation to the performance of the primary system is a limited cost to pay for providing communication opportunity to a large number of users in a CRSN. However, SINR of all the PRs is above a certain threshold level which shows that the proposed scheme minimally affect primary communications.

\section{Conclusion}

In this paper, we proposed a new concept of integrating ambient backscatter communications with the cognitive radio network, where STs are wirelessly powered by radio signals transmitted by the PT. Considering an underlay model for the secondary system, we proposed a scheme where STs choose between backscatter mode or harvestthen-transmit mode, based on the estimated interference that will be induced to PRs. The secondary system minimally affects the primary users' performance while coexisting with the primary network. Under the constraints of interference on PRs, the proposed scheme improves the average data rate of the secondary system in underlay CRSN. Furthermore, we evaluate the performance of the proposed scheme through system level simulations. Finally, the results are presented for the proposed scheme to show the improvement in the performance of the secondary system. We also highlight the slight degradation of the primary system which is a tradeoff for providing communication services to a large number of low power secondary users.

\section{Acknowledgements}

This work was supported by the National Research Foundation of Korea (NRF) grant funded by the Korean government (MSIP) (2014R1A5A1011478).

\section{Authors' contributions}

DM and MYC conceived the idea and designed the algorithm. STS helped in the evaluation of the proposed scheme. DM wrote the manuscript. KWC and $\mathrm{T}-\mathrm{J} \mathrm{L}$ helped in improving the algorithm and reviewing the manuscript. All authors read and approved the final manuscript.

\section{Competing interests}

The authors declare that they have no competing interests.

\section{Publisher's Note}

Springer Nature remains neutral with regard to jurisdictional claims in published maps and institutional affiliations.

\section{Author details}

${ }^{1}$ Department of Electrical and Computer Engineering, Sungkyunkwan University, 2066 Seobu-Ro, Jangan-Gu, 16419 Suwon, Gyeonggi-Do, South Korea. ${ }^{2}$ Department of Telecommunication Engineering, FICT, Balochistan University of Information Technology, Engineering and Management Sciences, Airport Road, Baleli, 87300 Quetta, Pakistan.

Received: 23 October 2017 Accepted: 6 February 2019

Published online: 21 February 2019

\section{References}

1. A. Ahmad, S. Ahmad, M.H. Rehmani, N.U. Hassan, A survey on radio resource allocation in cognitive radio sensor networks. IEEE Commun. Surv. Tutor. 17(2), 888-917 (2015)

2. G.I. Tsiropoulos, O.A. Dobre, M.H. Ahmed, K.E. Baddour, Radio resource allocation techniques for efficient spectrum access in cognitive radio networks. IEEE Commun. Surv. Tutor. 18(1), 824-847 (2016)

3. O.B. Akan, O.B. Karli, O. Ergul, Cognitive radio sensor networks. IEEE Netw. (23)4, 34-40 (2009)

4. M.E. Tanab, W. Hamouda, Resource allocation for underlay cognitive radio networks: a survey. IEEE Commun. Surv. Tutor. 19(2), 1249-1276 (2017)

5. V. Liu, A.N. Parks, V. Talla, S. Gollakota, D. Wetherall, J.R. Smith, Ambient Backscatter: Wireless Communication out of Thin Air. (Association for Computing Machinery (ACM), Hong Kong, 2013)

6. K. Han, K. Huang, Wirelessly powered backscatter communication networks: modeling, coverage and capacity. IEEE Trans. Wirel. Commun. 16(4), 2548-2561 (2017) 
7. B. Lyu, Z. Yang, G. Gui, Y. Feng, Optimal resource allocation policies for multi-user backscatter communication systems. Sensors. 16(12), 2016 (2016)

8. D.T. Hoang, D. Niyato, P. Wang, D.I. Kim, Opportunistic channel access and RF energy harvesting in cognitive radio networks. IEEE J. Sel. Areas Commun. 32(11), 2039-2052 (2014)

9. S. Bi, Y. Zeng, R. Zhang, Wireless powered communication networks: an overview. IEEE Wirel. Commun. 23(2), 10-18 (2016)

10. D.T. Hoang, D. Niyato, P. Wang, D.I. Kim, Z. Han, The Tradeoff Analysis in RF-Powered Backscatter Cognitive Radio Networks. Global Communications Conference (GLOBECOM). (Institute of Electrical and Electronics Engineers (IEEE), Washington, 2016)

11. K.H. Park, D. Munir, J.S. Kim, M.Y. Chung, Integrating RF-Powered Backscatter with Underlay Cognitive Radio Networks. (Institute of Electrical and Electronics Engineers (IEEE), Da Nang, 2017)

12. K. Hareesh, P. Singh, An Energy Efficient Hybrid Co-operative Spectrum Sensing Technique for CRSN. (Institute of Electrical and Electronics Engineers (IEEE), Kottayam, 2013)

13. Y. Lin, C. Wang, J. Wang, Z. Dou, A novel dynamic spectrum access framework based on reinforcement learning for cognitive radio sensor networks. Sensors. 16(10), 1675 (2016)

14. Y. Zhang, S. He, J. Chen, Y. Sun, X. Shen, Distributed sampling rate control for rechargeable sensor users with limited battery capacity. IEEE Trans. Wirel. Commun. 12(6), 3096-3106 (2013)

15. S. Gao, L. Qian, D.R. Vaman, Distributed energy efficient spectrum access in cognitive radio wireless ad hoc networks. IEEE Trans. Wirel. Commun. 8(10), 5202-5213 (2009)

16. J.A. Han, W.S. Jeon, D.G. Jeong, Energy-efficient channel management scheme for cognitive radio sensor networks. IEEE Trans. Veh. Techol. 60(4), 1905-1910 (2011)

17. L.R. Varshney, Transporting Information and Energy Simultaneously. (Institute of Electrical and Electronics Engineers (IEEE), Toronto, 2008)

18. S. He, J. Chen, F. Jiang, D.KY. Yau, G. Xing, Y. Sun, Energy provisioning in wireless rechargeable sensor networks. IEEE Trans. Mob. Comput. 12(10), 1931-1942 (2013)

19. Y. Zhang, S. He, J. Chen, Y. Sun, X.S. Shen, Distributed sampling rate control for rechargeable sensor users with limited battery capacity. IEEE Trans. Commun. 12(6), 3096-3106 (2013)

20. I. Yoon, M.Y. Jun, J. Semi, K.N. Dong, Adaptive sensing and compression rate selection scheme for energy harvesting wireless sensor networks. Int. J. Distrib. Sens. Netw. 13(6), 1-10 (2017)

21. Y. Wang, W. Lin, R. Sun, Y. Huo, Optimization of relay selection and ergodic capacity in cognitive radio sensor networks with wireless energy harvesting. Pervasive Mob. Comput. 22, 33-45 (2015)

22. S. Kalamkar, J. Jeyaraj, A. Banerjee, K. Rajawat, Resource allocation and fairness in wireless powered cooperative cognitive radio networks. IEEE Trans. Commun. 64(8), 3246-3261 (2016)

23. A.N. Parks, A. Liu, S. Gollakota, J.R. Smith, Turbocharging Ambient Backscatter Communication. (ACM SIGCOMM, Chicago, 2014)

24. B. Kellogg, V. Talla, S. Gollakota, J.R. Smith, Passive Wi-Fi: Bringing Low Power to Wi-Fi Transmissions. (The Advanced Computing Systems Association (USENIX), Santa Clara, 2016)

25. C. Boyer, S. Roy, Backscatter communication and RFID: coding, energy, and MIMO analysis. IEEE Trans. Commun. 62(3), 770-785 (2014)

26. B. Lyu, Z. Yang, G. Gui, H. Sari, Optimal time allocation in backscatter assisted wireless powered communication networks. Sensors. 17(6), 1258 (2017)

27. D.T. Hoang, D. Niyato, P. Wang, D.I. Kim, Optimal Time Sharing in RF-Powered Backscatter Cognitive Radio Networks. (Institute of Electrical and Electronics Engineers (IEEE), Paris, 2017)

28. R. Zhang, C.K. Ho, MIMO broadcasting for simultaneous wireless information and power transfer. IEEE Trans. Wirel. Commun. 12(5), 1989-2001 (2013)

29. W. Su, J.D. Matyjas, N.B. Stella, Active cooperation between primary users and cognitive radio users in heterogeneous ad-hoc networks. IEEE Trans. Signal Process. 60(4), 1796-1805 (2012)

30. L.B. Le, E. Hossain, Resource allocation for spectrum underlay in cognitive radio networks. IEEE Trans. Wirel. Commun. 7(12), 5306-5315 (2008)

31. ATSC digital television standard (1995). Advance Television System Committee (ATSC) Digital Television System Standards, ATSC Standard A/53, Online available at: https://www.atsc.org/standard/a53-atsc-digitaltelevision-standard/

32. D.K. Klair, K.-W. Chin, R. Raad, Asurvey survey and tutorial of RFID anti-collision protocols. IEEE Commun. Surv. Tutor. 12(3), 400-421 (2010)

\section{Submit your manuscript to a SpringerOpen ${ }^{\circ}$ journal and benefit from:}

- Convenient online submission

- Rigorous peer review

- Open access: articles freely available online

- High visibility within the field

- Retaining the copyright to your article

Submit your next manuscript at $\$$ springeropen.com 